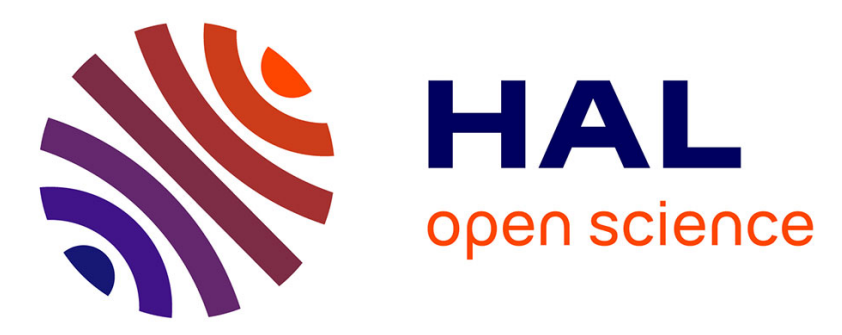

\title{
Mechanically induced Helfrich-Hurault effect in a confined lamellar system with finite surface anchoring
}

Riccardo de Pascalis

\section{To cite this version:}

Riccardo de Pascalis. Mechanically induced Helfrich-Hurault effect in a confined lamellar system with finite surface anchoring. 2019. hal-02163985

\section{HAL Id: hal-02163985 \\ https://hal.science/hal-02163985}

Preprint submitted on 24 Jun 2019

HAL is a multi-disciplinary open access archive for the deposit and dissemination of scientific research documents, whether they are published or not. The documents may come from teaching and research institutions in France or abroad, or from public or private research centers.
L'archive ouverte pluridisciplinaire HAL, est destinée au dépôt et à la diffusion de documents scientifiques de niveau recherche, publiés ou non, émanant des établissements d'enseignement et de recherche français ou étrangers, des laboratoires publics ou privés. 


\title{
Mechanically induced Helfrich-Hurault effect in a confined lamellar system with finite surface anchoring
}

\author{
Riccardo De Pascalis ${ }^{1 *}$ \\ ${ }^{1}$ Dipartimento di Matematica e Fisica 'E. De Giorgi', Università del Salento, Via per Arnesano, \\ 73100 , Lecce, Italy
}

\begin{abstract}
Soft lamellar phases confined between two parallel plates and subject to a dilatative strain can become unstable exhibiting periodic deformations patterns of the layers. By a variational energy approach, a critical threshold for the imposed finite strain is derived in the case of weak anchoring conditions. The potential, associated to the system, includes a two terms energy which accounts for the bending of the layers and the dilatation of the bulk as well as an anchoring potential. Classical results for strong anchoring at the walls are recovered. It is shown that weak anchoring conditions, can lead to a lower critical threshold of the field, similarly as it happens for the instability induced by a magnetic or an electric field normal to the layers. Nevertheless, in the limit of weak anchoring, the model reveals that this instability does not occur. Analytical formulas are provided which certainly encourage further experimental invetigations.
\end{abstract}

KEYWORDS: smectic-A liquids crystals, homeotropic alignment, Helfrich-Hurault effect, Weak anchoring, instability.

\section{Introduction}

Lamellar structures like cholesterics or smectics liquid crystals confined between two parallels plates and subject to external fields (electric, magnetic, as well as to a mechanically deformation) can, under a certain critical threshold, become unstable and buckle into a new different configuration [1, 2, 3, 4,

Cholesteric liquid crystals samples subject to a magnetic or electric field applied normally to its layers, tend to reorients them along the normal while the molecules anchored at the boundary walls do not allow the adjacent layers to freely rotate. In the early seventies, Helfrich [5] and Hurault 6] firstly observed that this competition can lead to periodic undulations of the layers orientation. This instability is nowadays still known in literature as Helfrich-Hurault effect 11. Theoretical predictions for this instability and for an infinite sample can be found in [1, 7] while theoretical results extended to finite samples of Smectic-A liquid crystals, if subject to both a uniform pressure and a magnetic field, can be found in [8] and if subject to an electric field in [9, 10. Further theoretical results for the Helfrich-Hurault instability induced by a magnetic field but for Smectic-C liquid crystals can be found in [11, 12].

Ishikawa and Lavrentovich [13] observed the undulations of the layered systems of cholesteric stripe phase with a macroscopic supramicron periodicity induced by an in-plane magnetic field normal to the layers. Few years later, Senyuk et al. [14] observed this instability for a confined cholesteric liquid crystals sample subject to an electrical field applied along the normal to the layers. Both studies [13, 14] emphasize that a displacement of the layers immediately above the instability threshold is much larger than the values expected from the previous classical theories. They were able to describe their experimental data by including a finite anchoring potential to the wall, demonstrating (for the $2 \mathrm{D}$ case in [13] and for the extended 3D case in [14]), that the undulations depend from the molecules anchoring at the wall. Moreover they deduced, qualitatively and quantitatively, that the finite strong conditions can decrease the critical threshold applied field allowing larger displacements of the layers.

\footnotetext{
${ }^{*}$ Author for correspondence: riccardo.depascalis@unisalento.it
} 
Undulations of the layers can be also caused by a dilatative mechanical applied deformation [15, 16, 17, 18, 19, 20, where layers, in order to increase their effective thickness due to the dilatation, tend to tilt and to balance the stretch imposed to the sample which tends to separate the boundary walls. All these studies are however confined to the case of strong anchoring conditions. In particular [18, 19] explored the case of non-linear undulations where the instability show a transition from sinusoidal to a chevron structure. Napoli and Nobili [20, extended the classical results valid for infinitesimal imposed strain (see equation (40) ) to the most general case valid for an imposed finite dilatative strain (see equation (39) 1 ), capable therefore to cover cases were the specimen thickness $d$ can be comparable to the characteristic length $\lambda$. Analogous observed instabilities are reported in [21, 22. The former refers to active cholesteric liquid crystals where buckling can be induced by both extensile or contractile applied stresses. Whilst the latter concerns freely floating smectic liquid crystalline films [22] where spontaneous wrinkling can appear in order to compensate lateral compressions.

Here, the Helfrich-Hurault effect is analysed for an infinite smectic-A liquid crystals sample which exhibits homeotropic alignments of the layers and it is subjected to a dilatative finite applied strain along the normal to the layers when molecules are weakly anchored at the walls. By a variational energy approach, a critical strain $\gamma_{\mathrm{c}}$ at which buckling can occur is investigated for the symmetric anchoring case. The energy associated to the system includes a classical two terms energy, with one term associated to the bending of the layers and the other associated to the dilatation of the bulk (see equation (11)), as well as a Rapini and Papoular type anchoring potential 23 at the walls (see equation (16)). Classical results for strong anchoring conditions are recovered [1, 15, 16, noticing that the derived model is valid to describe both the infinitesimal and the finite applied strain case (see [20] for further details). It is noteworthy, for example, the experimental findings on the cholesteric fingerprint texture with a macroscopic $\approx 10 \mathrm{~mm}$ periodicity which profile deviates from the classic pattern predicted by the linear elastic theory but fits well with the nonlinear theory of dislocations [24, 25].

Furthermore, in analogy with a sample subject to a magnetic [13] or eletric field 14 normal to the layers, respectively, here, we show also that a similar result is still valid, i.e. finite anchoring conditions lead to the lower critical threshold field. Nevertheless, the equations derived from this model reveal that, in the limit of weak anchoring, the type of instability here considered does not occur. An analytical expression for the anchoring strength bound in terms of $\eta=\lambda / d$, beyond which the instability is not predicted from this model, is provided. Worth to note that this feature is also exhibited from the critical field derived in 13 . for the sample subject to an external magnetic field normal to the layers. Furher experimental investigations are therefore encouraged in order to explore the limit case of weak anchoring conditions. Remarkably what it has been observed for cholesteric liquid crystal samples with short pitch, which can behave as a layered smectic-A liquid crystals, and for which under an applied electric field sufficiently larger than the anchoring energy, the non linear undulations can be transformed to a system of defects [26, 27].

The paper is organized as follows: Sec. 2 introduces the geometry of the sample, the applied deformation field as well as the assumptions on the energy associated to it. In Sec. 3, in order to compute the critical threshold, the Euler-Lagrange equation with boundary conditions at the walls are derived by a variational approach. These equations are then specialized to the symmetric anchoring in Sec. 4. where analytical formulas for the whole critical field are derived. Importantly, it is shown that the limit for strong anchoring conditions is recovered while the instability does not occur for very weak anchoring. In this last section, we provide this analytical cut-off critical bound as well as a general discussion of the obtained results.

\section{The model}

\subsection{Geometric preliminaries}

Smectic- $A$ liquid crystals can be described by isosurfaces $\varphi_{k}$ ( $k$ labels the layer) defined by

$$
\varphi(\mathbf{x}, k)=0
$$


where $\mathbf{x}$ denotes the current vector position of a point on $\varphi_{k}$. In particular, in the undeformed state such surfaces are parallel planes described by the relationship

$$
\varphi_{0}(\mathbf{X}, k)=\mathbf{N} \cdot \mathbf{X}-k \ell_{0},
$$

where $\mathbf{X}=X \mathbf{E}_{1}+Z \mathbf{E}_{3}$ is a position vector of a point written in its undeformed coordinates, $\mathbf{N}$ denotes the unit normal vector $\mathbf{N} \equiv \mathbf{E}_{3}$, while $\ell_{0}$ represents the distance between the considered plane and the reference plane $Z=0$, being $O(X, Y, Z)$ a Cartesian reference frame (see Fig. 1 for a schematic representation of the sample in the undeformed configuration). The operator '.' denotes the inner product.

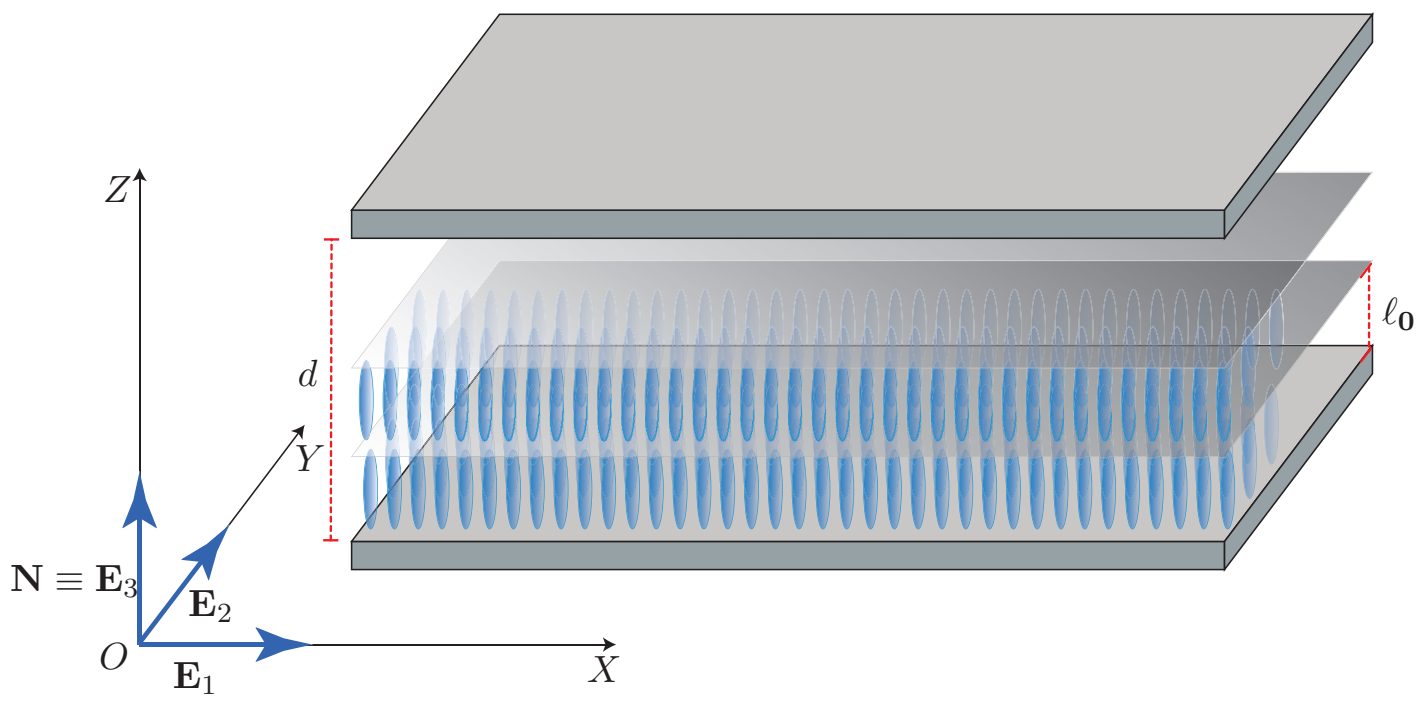

Figure 1: A sample of a smectic-A liquid crystal in the undeformed configuration (no scale is implied).

Let us consider the invertible transformation $\mathbf{x}=\chi(\mathbf{X})$, which maps the undeformed configuration into the distorted one described, in a Cartesian coordinate system, by $O(x, y, z)$. By using its inverse $\mathbf{X}=\chi^{-1}(\mathbf{x})$, equation (2) can be rewritten as

$$
\varphi(\mathbf{x}, k)=\mathbf{N} \cdot \chi^{-1}(\mathbf{x})-k \ell_{0} .
$$

Let us denote by $\mathbf{F}$ the deformation gradient of the transformation $\chi$ which, in components form, is given by $F_{i j}=(\operatorname{Gradx})_{i j}=\partial x_{i} / \partial X_{j},(i, j=1,2,3)$. By taking the spatial gradient of equation (3i) with respect to $\mathbf{x}$ coordinates and by application of the chain rule,

$$
\nabla \varphi=\frac{\partial \varphi}{\partial \mathbf{x}}=\frac{\partial \varphi_{0}}{\partial \mathbf{X}} \frac{\partial \mathbf{X}}{\partial \mathbf{x}}=\mathbf{F}^{-\mathrm{T}} \frac{\partial \varphi_{0}}{\partial \mathbf{X}}
$$

where the superscript ' $-\mathrm{T}$ ' denotes the transpose of the inverse, so that

$$
\nabla \varphi=\mathbf{F}^{-\mathrm{T}} \mathbf{E}_{3} .
$$

A cell of material, which is in the homeotropic alignment, between two parallel planes $Z=0$ and $Z=d$ is subjected to a following deformation

$$
\mathbf{x}=\mathbf{X}+U(X, Z) \mathbf{E}_{3},
$$

which, in the $X Z$-plane, gives

$$
\mathbf{F}=\left(\begin{array}{ccc}
1 & 0 & 0 \\
0 & 1 & 0 \\
\partial_{X} U & 0 & 1+\partial_{Z} U
\end{array}\right), \quad \mathbf{F}^{-\mathrm{T}}=\left(\begin{array}{ccc}
1 & 0 & \frac{-\partial_{X} U}{1+\partial_{Z} U} \\
0 & 1 & 0 \\
0 & 0 & \frac{1}{1+\partial_{Z} U}
\end{array}\right)
$$


where $\partial_{X}$ and $\partial_{Y}$ denote the partial derivatives with respect to $X$ and $Y$, respectively. From (5) and (7) 2 it follows that

$$
|\nabla \varphi|^{2}=\frac{1+\left(\partial_{X} U\right)^{2}}{\left(1+\partial_{Z} U\right)^{2}}
$$

Let consider the displacement $U$ as a small perturbation superposed to a finite homogeneous displacement, i.e.

$$
U(X, Z)=\gamma Z+\epsilon u(X, Z),
$$

where $\gamma>0$ is a strain along the $Z$-direction and $\epsilon$ represents a small perturbative dimensionless positive parameter

$$
\epsilon=\sqrt{\frac{\gamma}{\gamma_{c}}-1}
$$

being $\gamma_{\mathrm{c}}$ the critical strain at which buckling occurs. Note that for $\epsilon=0$ the $\ell_{0}$ distance changes to a current distance $\ell$ given by $\ell=(1+\gamma) \ell_{0}$.

\subsection{Distortion energy density}

When a deformation field is imposed, the layers can undergo a static distortion with respect to their natural configuration. The free energy related to this distortion is the elastic energy density [28]

$$
f_{\mathrm{e}}=\frac{K}{2}(\operatorname{div} \mathbf{n})^{2}+\frac{B}{2}\left(\frac{1}{|\nabla \varphi|}-1\right)^{2}
$$

where $K$ and $B$ are two positive constants called the bending stiffness and the compression modulus, respectively, and where $\mathbf{n}$ is the unit normal vector in the current configuration,

$$
\mathbf{n}=\frac{\nabla \varphi}{|\nabla \varphi|}
$$

The first term of (11) penalizes the layer bending, since $\frac{1}{2}$ div $\mathbf{n}$ represents the mean curvature of the layer surface. The second term represents the energy related to the dilation/compression of the layers thickness.

By application of the chain rule, we obtain

$$
\operatorname{div} \mathbf{n}=\operatorname{Grad} \mathbf{n} \cdot \mathbf{F}^{-\mathrm{T}}=\operatorname{Tr}\left(\mathbf{F}^{-1} \operatorname{Grad} \mathbf{n}\right)=-\epsilon \partial_{X X} u+\mathcal{O}\left(\epsilon^{2}\right),
$$

while

$$
\frac{1}{|\nabla \varphi|}-1=\gamma+\epsilon \partial_{Z} u-\frac{\epsilon^{2}}{2}(1+\gamma)\left(\partial_{X} u\right)^{2}
$$

so that, up to the second order in $\epsilon$, the elastic energy density (11) can be written as

$$
f_{\mathrm{e}}=\frac{1}{2} K\left(\partial_{X X} u\right)^{2} \epsilon^{2}+\frac{B}{2}\left(\gamma^{2}+2 \gamma \epsilon \partial_{Z} u+\epsilon^{2}\left(\left(\partial_{Z} u\right)^{2}-\gamma(\gamma+1)\left(\partial_{X} u\right)^{2}\right)\right)+\mathcal{O}\left(\epsilon^{3}\right) .
$$

\subsection{Anchoring potential}

According to the Rapini and Papoular formula [23, we assume that, at the walls, $\mathbf{n}$ prefers to align along the $\mathbf{E}_{3}$ direction. Consequently, the anchoring energy takes the form

$$
f_{\mathrm{a}}=\frac{1}{2}\left[w_{-}\left(\mathbf{n} \cdot \mathbf{E}_{1}\right)_{Z=0}^{2}+w_{+}\left(\mathbf{n} \cdot \mathbf{E}_{1}\right)_{Z=d}^{2}\right]
$$

where $w_{ \pm}$are two positive constants, and $\mathbf{n}$ is the unit normal vector (12) in the deformed configuration, given by

$$
\mathbf{n}=\frac{1}{\sqrt{1+\epsilon^{2} u_{X}^{2}}}\left(-\epsilon u_{X} \mathbf{E}_{1}+\mathbf{E}_{3}\right) .
$$




\subsection{Non-dimensionalization}

The quantity $\lambda=\sqrt{K / B}$ defines a characteristic length of the material which is of the order of the layers thickness while $K / w$, named the extrapolation length, is the measure for the relevance of the competing elastic distortion versus the anchoring induced order. Let define the non-dimensional parameters

$$
\xi=\frac{X}{d}, \quad \zeta=\frac{Z}{d}, \quad v=\frac{u}{d}, \quad \eta=\frac{\lambda}{d}, \quad \beta_{ \pm}=\frac{d}{K} w_{ \pm},
$$

which allow to rewrite the energies densities (11) and (16) in a dimensionless form,

$$
\phi_{\mathrm{e}}=f_{\mathrm{e}} \frac{d^{2}}{K}=\frac{1}{2}\left(v_{\xi \xi}\right)^{2} \epsilon^{2}+\frac{1}{2 \eta^{2}}\left(\gamma^{2}+2 \gamma \epsilon v_{\zeta}+\epsilon^{2}\left(v_{\zeta}^{2}-\gamma(\gamma+1) v_{\xi}^{2}\right)\right)+\mathcal{O}\left(\epsilon^{3}\right)
$$

and

$$
\phi_{\mathrm{a}}=f_{\mathrm{a}} \frac{d}{K}=\frac{1}{2}\left[\beta_{-} v_{\xi}^{2}(\xi, 0)+\beta_{+} v_{\xi}^{2}(\xi, 1)\right] \epsilon^{2}+\mathcal{O}\left(\epsilon^{4}\right),
$$

respectively. The quantities $\beta_{-}$and $\beta_{+}$given in (18) measure the strength of the anchoring at the wall $\zeta=0$ and $\zeta=1$, respectively (see [1] for further details). Note that the strong planar anchoring conditions, where layers are clamped at the walls, are recovered in the limit $\beta_{ \pm} \rightarrow \infty$. On the contrary, the conditions $\beta_{ \pm}=0$ expresses free anchoring conditions and, in this case, the layers are simply supported at the walls.

\section{Critical threshold}

We assume that the perturbative displacement field is separable in $\zeta$ and $\xi$ by an amplitude unknown $\zeta$-dependent function and a periodic cosine $\xi$-dependent function [2, 20, i.e.

$$
v(\xi, \zeta)=a(\zeta) \cos \left(q_{\xi} \xi\right)
$$

with $q_{\xi}$ representing a dimensionless wave number along the $x$ direction. By integrating the total energy density $\phi_{\mathrm{e}}+\phi_{\mathrm{a}}$ with respect to $\xi$ and averaging it over the period $T=2 \pi / q_{\xi}$ and then by integrating it with respect to the $\zeta$ variable in $[0,1]$, we obtain the total potential of the system

$$
\Phi=\frac{\gamma^{2}}{2 \eta^{2}}+\frac{\epsilon^{2}}{4 \eta^{2}} \int_{0}^{1} \Psi\left(a, a^{\prime}\right) \mathrm{d} \zeta+\frac{\epsilon^{2}}{4} q_{\xi}^{2}\left(\beta_{-} a^{2}(\zeta)_{\mid \zeta=0}+\beta_{+} a^{2}(\zeta)_{\mid \zeta=1}\right)+\mathcal{O}\left(\epsilon^{3}\right)
$$

where $\Psi\left(a, a^{\prime}\right)=\Lambda a^{2}+a^{\prime 2}$ with the prime denoting the differentation of a function with respect to its argument and

$$
\Lambda=q_{\xi}^{2}\left(q_{\xi}^{2} \eta^{2}-\gamma_{\mathrm{c}}\left(\gamma_{\mathrm{c}}+1\right)\right) .
$$

Equilibrium configurations are stationary points of the free energy functional $\Phi$. By considering a certain test function $\alpha$, we impose

$$
\Phi^{\prime}(a)(\alpha)=\frac{\mathrm{d}}{\mathrm{d} t} \Phi(a+t \alpha)_{\mid t=0}=0,
$$

and at second order in $\epsilon$, after carrying some straightforward algebra and from the arbitrariness of the function $\alpha$, we deduce the Euler-Lagrange equation for $a$

$$
a^{\prime \prime}-\Lambda a=0,
$$

which must also satisfies the following boundaries conditions (BCs)

$$
\begin{array}{ll}
a^{\prime}-\beta_{-} \eta^{2} q_{\xi}^{2} a=0, & \text { at } \zeta=0, \\
a^{\prime}+\beta_{+} \eta^{2} q_{\xi}^{2} a=0, & \text { at } \zeta=1 .
\end{array}
$$

Note that $\gamma$ positive assures $\Lambda$ negative. Thus, the most general solution of the equation (25) can be written as combination of sine and cosine functions in the following manner

$$
a(\zeta)=C_{1} \cos \left(q_{\zeta} \zeta\right)+C_{2} \sin \left(q_{\zeta} \zeta\right),
$$


where $q_{\zeta}$ denotes a dimensionless wave number along the $z$ direction and where $C_{1}, C_{2}$ are two integration constants. BCs (26)-(27) allow to obtain one of these constants as well as the relation imposed to $q_{\zeta}$ and $q_{\xi}$, i.e.

$$
C_{1}=\frac{C_{2} q_{\zeta}}{\beta_{-} \eta^{2} q_{\xi}^{2}}, \quad f\left(q_{\zeta}, q_{\xi}\right)=0
$$

where

$$
f\left(q_{\zeta}, q_{\xi}\right)=\left(\beta_{-} \beta_{+} \eta^{4} q_{\xi}^{4}-q_{\zeta}^{2}\right) \sin q_{\zeta}+\left(\beta_{-}+\beta_{+}\right) \eta^{2} q_{\xi}^{2} q_{\zeta} \cos q_{\zeta}
$$

\section{Symmetric anchoring: results and discussion}

In the symmetric anchoring case $\beta_{-}=\beta_{+}=\beta$, in order to take into account the imposed symmetry at the walls as well as to cover results for strong anchoring conditions ([13, 20]), we can further assume $a$ be of the form

$$
a(\zeta)=A \cos \left[q_{\zeta}\left(\frac{1}{2}-\zeta\right)\right],
$$

where the amplitude $A$ is a constant to be determined. Note that BCs (26)-(27) reduce now to one independent relation

$$
q_{\xi}^{2}=\frac{q_{\zeta}}{\beta \eta^{2}} \tan \left(q_{\zeta} / 2\right)
$$

which has to be satisfied with the constraint given by imposing $a$ in (31) be a solution of (25)

$$
q_{\zeta}^{2}=-\Lambda \text {. }
$$

By the assumption on the solution made in (21) and (31), respectively, the minimization of the total energy (22) with respect to $q_{\xi}$ allows to determine the critical wave number

$$
q_{\xi}^{2}=\frac{\gamma_{\mathrm{c}}\left(\gamma_{\mathrm{c}}+1\right)}{2 \eta^{2}}-\beta q_{\zeta} \frac{1+\cos q_{\zeta}}{q_{\zeta}+\sin q_{\zeta}},
$$

which, combined with (32) and (33), gives rise a nonlinear equation for the wave number $q_{\zeta}$

$$
\tan ^{2}\left(\frac{q_{\zeta}}{2}\right)+\beta^{2} \eta^{2} \frac{\sin q_{\zeta}-q_{\zeta}}{\sin q_{\zeta}+q_{\zeta}}=0 .
$$

In terms of existence of solutions for $q_{\zeta}$, equation (35) exhibits a different behaviour in the limit of strong anchoring from that of finite weak anchoring. Whilst it guarantees solutions $q_{\zeta}$ for any large value of the strength parameter $\beta$, on the contrary (35) reveals a lower $\beta$ cut-off bound, which clearly depends on $\eta$, and beyond which $q_{\zeta}$ solutions do not exist. Indeed, developing $\beta$ in powers of $q_{\zeta}$ up to order two, it must hold

$$
\beta=\frac{\sqrt{3}}{\eta}+\frac{q_{\zeta}^{2}}{5 \sqrt{3} \eta}+\mathcal{O}\left(q_{\zeta}^{4}\right),
$$

which gives the lower expected $\beta$ (at a fixed $\eta$ ) for the occurrence of the instability as $q_{\zeta} \rightarrow 0$. Note that equation (35) is the equivalent of equation (5) in [13] when a sample of cholesteric is subjected to a magnetic field normal to the layers.

By replacing the $\beta$ 's expression from (35) into (32) and (33) and after a straightforward algebra, we can derive the expression for $\gamma_{\mathrm{c}}$ and $q_{\xi}$ in terms of the computed critical $q_{\zeta}$ and the parameter $\eta$

$$
\gamma_{\mathrm{c}}=\frac{1}{2}\left(-1+\sqrt{1+8 \eta q_{\zeta}^{2} \sqrt{\frac{1}{q_{\zeta}^{2}-\sin ^{2} q_{\zeta}}}}\right), \quad q_{\xi}^{2}=\frac{q_{\zeta}}{\eta} \sqrt{\frac{q_{\zeta}-\sin q_{\zeta}}{q_{\zeta}+\sin q_{\zeta}}} .
$$

respectively. For $q_{\zeta} \approx 0$ they become

$$
\gamma_{\mathrm{c}}=\frac{1}{2}(-1+\sqrt{1+8 \sqrt{3} \eta})+\frac{2 \eta q_{\zeta}^{2}}{5 \sqrt{3+24 \sqrt{3} \eta}}+\mathcal{O}\left(q_{\zeta}^{4}\right), \quad q_{\xi}^{2}=\frac{q_{\zeta}^{2}}{2 \sqrt{3} \eta}+\mathcal{O}\left(q_{\zeta}^{4}\right),
$$


respectively, which allows to find easily their bounds values as $q_{\zeta} \rightarrow 0$.

According to the experimental setup carried in [13], let consider $\lambda=2.9 \mu \mathrm{m}$ and $d=1.7 \mathrm{~mm}$ which set $\eta=\eta_{\mathrm{IL}} \approx 1.7 \cdot 10^{-3}$. Figure 2 shows the predictions for the critical waves numbers $q_{\zeta}$ and $q_{\xi}$, against the scaled strength anchoring $\bar{\beta}=10^{-3} \beta$ and predicted by (35) and (37) 2 , respectively. Note, from Fig. 2a, that the classical limit $q_{\zeta} \rightarrow \pi$ of strong anchoring conditions is recovered, [15, 13, 20]. Indeed as $\beta \rightarrow \infty$ the relationship (32) imposes $q_{\zeta} \rightarrow k \pi$ (with $k$ a non zero integer number) which nontrivial critical minimum threshold is obviously attained for $k=1$, i.e. at $q_{\zeta}=\pi$. Consequently, in this limit, from (37) the critical strain $\gamma_{\mathrm{c}}$ and critical wave number $q_{\xi}$ reduce to

$$
\gamma_{\mathrm{c}}=-\frac{1}{2}+\frac{1}{2} \sqrt{1+8 \pi \eta}, \quad q_{\xi}=\sqrt{\frac{\pi}{\eta}},
$$

in agreement with [20] where it is remarked that, for $\eta \ll 1$, (39) 1 reduces to a classical linear result $([1,15,16])$

$$
\gamma_{0}=2 \pi \eta
$$

Instead, for very low $\beta$, curves in Fig. $2 \mathrm{a}$ follow the law (36) and those in Fig. 2b the law (37) 2 .

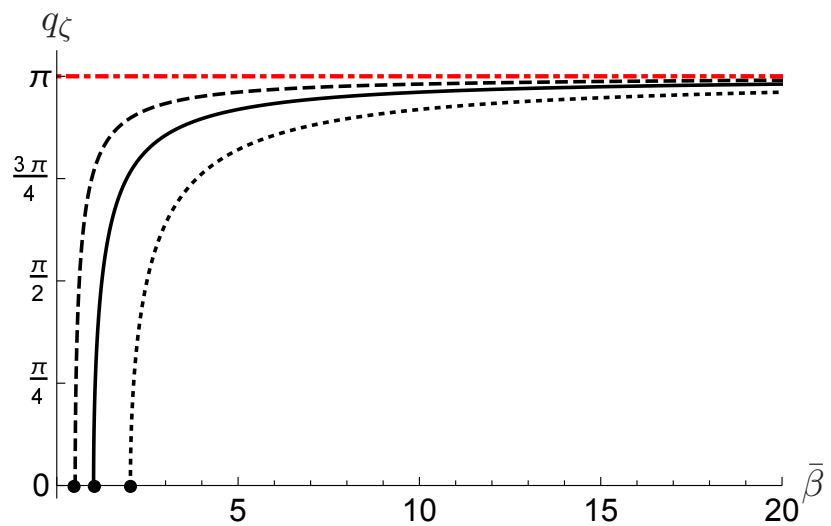

(a)

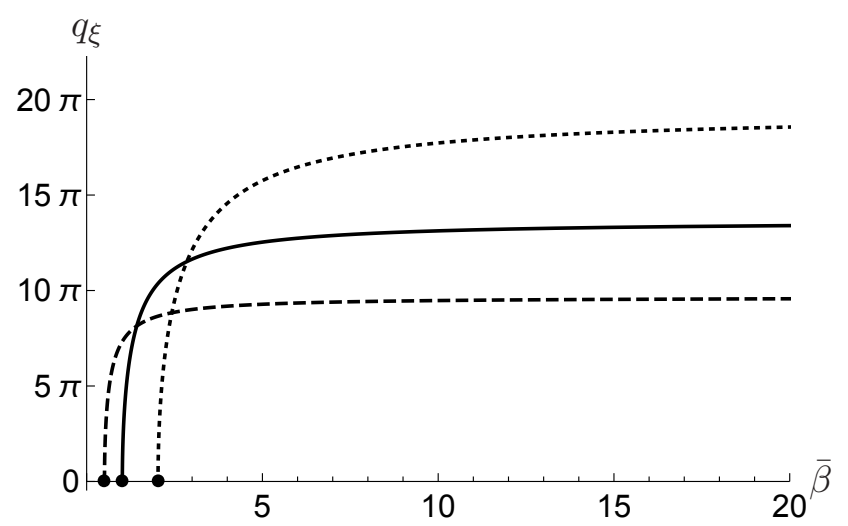

(b)

Figure 2: The critical wave numbers when $\eta=\eta_{\mathrm{IL}}$ (solid line), $\eta=1 / 2 \eta_{\mathrm{IL}}$ (dotted line) and $\eta=2 \eta_{\mathrm{IL}}$ (dashed line) against $\bar{\beta}=10^{-3} \beta$ and predicted by (35) in (a) and (37) 2 in (b), respectively.

Fig. 3 shows several predictions of $\gamma_{\mathrm{c}} / \eta$ versus $\bar{\beta}$, for $\eta$ in a range $\left[\eta_{\mathrm{IL}}, 60 \eta_{\mathrm{IL}}\right]$ being $\eta=60 \eta_{\mathrm{IL}} \approx 0.1$. Firstly, note that all curves are monotonic in $\beta$, confirming here the analogous result given in [13] and [14, i.e. finite anchoring at the walls favours (compared to the case of strong anchoring) the instability. Nevertheless, it is worth to note that all curves arise from a lower cut-off bound (represented in the graph by a red dashed curve obtained in the continuous limit $q_{\zeta} \rightarrow 0$ according to (36) and (38) 1 ). Thus, the model suggests that very weak anchoring of molecules at the boundary walls might not be sufficient for the system to compensate the effect instead observed for stronger anchoring conditions and instability is not favoured. Finally, as $\bar{\beta} \rightarrow \infty$, all the curves tend asymptotically to a different limit $\gamma_{\mathrm{c}} / \eta$ according to (39) in disagreement with the classical limit $\gamma_{0} / \eta \rightarrow 2 \pi$ given by using (40).

The derived model accounts for an instability of the Helfrich-Hurault type induced from an incremental deformation superposed to a finite homogeneous dilatation of the cell along the normal to the layers. The obtained results are therefore valid to predict the instabilities occurring in the linear case $\eta \ll 1$ as well as to recover the case for larger $\eta$. In the particular case when strong anchoring at the walls is applied, Napoli et al., in [20], showed (see their Fig. 2) the discrepancy between $\gamma_{0}$ and $\gamma_{\mathrm{c}}$ versus $\eta$, which becomes significant for cell thickness $d$ comparable to the characteristic length $\lambda$. In fact considering a 1-stearoyl-2oleoyl-3-sn-phospatidylcholine sample of thickness $d=40 \AA$ and $\lambda \approx 4.47 \AA$ ([29, 30, 31]), which implies $\eta \approx 0.09$, they showed a $29 \%$ of difference between the classical $\gamma_{0}$ and $\gamma_{\mathrm{c}}$. According to the parameters set deduced in [14] for a cholesteric liquid crystal confined between two parallel planes subject to an electric field applied along the normal to the layers, $B \approx 10 \mathrm{~J} / \mathrm{m}^{3}, K=5.8 \mathrm{pN}$ and $d \approx 60 \mu \mathrm{m}$ which imply $\eta=\eta_{\mathrm{SSL}} \approx 0.013$. In this latter case and for strong anchoring conditions the 
differences between $\gamma_{\mathrm{c}}$ and $\gamma_{0}$ woud be of the $8 \%$. Although it is still reasonable small, this disagreement would there be more consistent for weaker anchoring conditions, whereas, it has been observed larger layers displacements (see [14]). The present model would therefore predict more accurately also those most general cases.

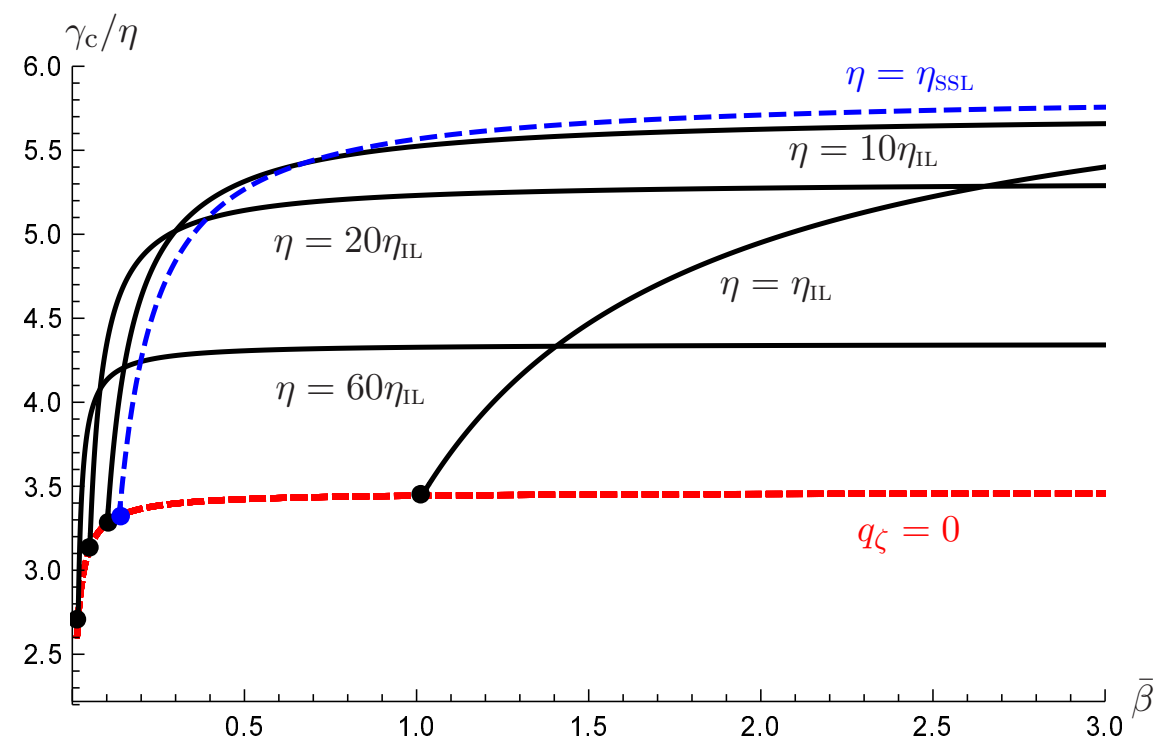

Figure 3: Predictions of $\gamma_{c}$ scaled by $\eta$ against $\bar{\beta}=10^{-3} \beta$ for several values of $\eta$. The red dashed curve show the lower cut-off bound for $q_{\zeta}=0$.

To conlude the analysis, at the critical threshold, the incremental deformation field, can therefore, be written as

$$
v(\xi, \zeta)=A \cos \left[q_{\zeta}\left(\frac{1}{2}-\zeta\right)\right] \cos \left(q_{\xi} \xi\right),
$$

with $q_{\zeta}$ given as solution of (35) and $q_{\xi}$ given by (37) 2 and where the amplitude $A$ is still an unknown of the problem. Following the proposed scheme in [13] and [32, in order to compute $A$, we impose to the total energy (22) a perturbed strain $\gamma=\gamma_{c}\left(1+\epsilon^{2}\right)$ and we retain it up to its fourth order term in $\epsilon$,

$$
\begin{aligned}
& \Phi=\frac{\gamma_{\mathrm{c}}^{2}}{2 \eta^{2}}+ \frac{\epsilon^{2}}{4 \eta^{2}}\left[\int_{0}^{1}\left(a^{\prime 2}+\Lambda a^{2}\right) \mathrm{d} \zeta+4 \gamma_{\mathrm{c}}^{2}+\eta^{2} \beta q_{\xi}^{2}\left(a^{2}(0)+a^{2}(1)\right)\right] \\
&+\frac{\epsilon^{4}}{64 \eta^{2}}\left[\int_{0}^{1} q_{\xi}^{2} a^{2}\left(3 q_{\xi}^{2} a^{2}\left(\gamma_{\mathrm{c}}\left(4 \gamma_{\mathrm{c}}+5\right)-4 \eta^{2} q_{\xi}^{2}+1\right)-4\left(a^{\prime 2}+4 \gamma_{\mathrm{c}}\left(2 \gamma_{\mathrm{c}}+1\right)\right)\right) \mathrm{d} \zeta\right. \\
&\left.+\frac{\gamma_{\mathrm{c}}^{2}}{2}-12 \eta^{2} q_{\xi}^{4} \beta\left(a^{4}(0)+a^{4}(1)\right)\right]+\mathcal{O}\left(\epsilon^{5}\right) .
\end{aligned}
$$

The minimization of the fourth order term in $\epsilon$ of (42) with respect to $A$, allows to find the unknown amplitude $A \neq 0$ which is a solution of the following second order equation

$$
\mathcal{C} A^{2}+\mathcal{D}=0
$$

with

$$
\mathcal{C}=\frac{1}{64}\left(2 q_{\zeta}\left(-4 q_{\zeta}^{2}+2 q_{\zeta} \sin \left(2 q_{\zeta}\right)+9 \Gamma q_{\xi}^{2}\right)+3 \Gamma q_{\xi}^{2}\left(8 \sin q_{\zeta}+\sin \left(2 q_{\zeta}\right)\right)\right)-6 \beta\left(\eta^{2} q_{\zeta} q_{\xi}^{2} \cos ^{4}\left(\frac{q_{\zeta}}{2}\right)\right),
$$

and

$$
\mathcal{D}=-\gamma_{\mathrm{c}}\left(1+2 \gamma_{\mathrm{c}}\right)\left(q_{\zeta}+\sin q_{\zeta}\right)
$$

where $\Gamma=1+\gamma_{\mathrm{c}}\left(5+4 \gamma_{\mathrm{c}}\right)-4 \eta^{2} q_{\xi}^{2}$. Finally, in the limit of strong anchoring $\beta \rightarrow \infty$ the results given in 20]

$$
A= \pm 4 \sqrt{\frac{2}{\pi}} \sqrt{\frac{\eta(1+8 \pi \eta-\sqrt{1+8 \pi \eta})}{9+64 \pi \eta+9 \sqrt{1+8 \pi \eta}}}
$$


are also recovered, which show that for $\eta \ll 1$, the amplitude $A$ can be approximated by

$$
A= \pm \frac{8}{3} \eta \mp \frac{128 \pi}{27} \eta^{2} \pm \frac{1360 \pi^{2}}{81} \eta^{3}+\mathcal{O}\left(\eta^{4}\right) .
$$

\section{Acknowledgments}

The author is grateful to Gaetano Napoli for fruitful discussions on this topic.

\section{References}

[1] P. de Gennes and J. Prost, The Physics of Liquid Crystals. Clarendon Press, Oxford, 2nd ed., 1993.

[2] I. W. Stewart, The static and dynamic continuum theory of liquid crystals, vol. 17. Taylor and Francis, London, 2004.

[3] P. Oswald and P. Pieranski, Smectic and columnar liquid crystals: concepts and physical properties illustrated by experiments. CRC Press, 2005.

[4] A. Jákli, O. D. Lavrentovich, and J. V. Selinger, "Physics of liquid crystals of bent-shaped molecules," Rev. Mod. Phys., vol. 90, p. 045004, Nov 2018.

[5] W. Helfrich, "Deformation of cholesteric liquid crystals with low threshold voltage," Applied Physics Letters, vol. 17, no. 12, pp. 531-532, 1970.

[6] J. P. Hurault, "Static distortions of a cholesteric planar structure induced by magnetic or ac electric fields," The Journal of Chemical Physics, vol. 59, no. 4, pp. 2068-2075, 1973.

[7] S. Chandrasekhar, Liquid Crystals. Cambridge University Press, 2 ed., 1992.

[8] I. W. Stewart, "Layer undulations in finite samples of smectic-a liquid crystals subjected to uniform pressure and magnetic fields," Phys. Rev. E, vol. 58, pp. 5926-5933, Nov 1998.

[9] G. Bevilacqua and G. Napoli, "Reexamination of the helfrich-hurault effect in smectic-a liquid crystals," Physical Review E, vol. 72, no. 4, p. 041708, 2005.

[10] G. Bevilacqua and G. Napoli, "The slight distortions induced by an electrostatic field on finite samples of smectic-a liquid crystals," Molecular Crystals and Liquid Crystals, vol. 436, no. 1, pp. 1271081, 2005.

[11] I. W. Stewart, "Layer distortions induced by a magnetic field in planar samples of smectic c liquid crystals," Liquid Crystals, vol. 30, no. 8, pp. 909-920, 2003.

[12] A. J. Walker and I. W. Stewart, "Layer undulations in a smectic c liquid crystal with weak anchoring," Journal of Physics A: Mathematical and Theoretical, vol. 40, pp. 11849-11861, sep 2007.

[13] T. Ishikawa and O. D. Lavrentovich, "Undulations in a confined lamellar system with surface anchoring," Phys. Rev. E, vol. 63, p. 030501, Feb 2001.

[14] B. I. Senyuk, I. I. Smalyukh, and O. D. Lavrentovich, "Undulations of lamellar liquid crystals in cells with finite surface anchoring near and well above the threshold," Phys. Rev. E, vol. 74, p. 011712, Jul 2006.

[15] N. A. Clark and R. B. Meyer, "Strain-induced instability of monodomain smectic a and cholesteric liquid crystals," Applied Physics Letters, vol. 22, no. 10, pp. 493-494, 1973.

[16] M. Delaye, R. Ribotta, and G. Durand, "Buckling instability of the layers in a smectic-a liquid crystal," Physics Letters A, vol. 44, no. 2, pp. 139 - 140, 1973. 
[17] R. Ribotta and G. Durand, "Mechanical instabilities of smectic-a liquid crystals under dilative or compressive stresses," Journal de Physique, vol. 38, no. 2, pp. 179-204, 1977.

[18] S. J. Singer, "Layer buckling in smectic-a liquid crystals and two-dimensional stripe phases," Physical Review E, vol. 48, no. 4, p. 2796, 1993.

[19] P. Oswald, J.-C. Géminard, L. Lejvcek, and L. Sallen, "Nonlinear analysis of stripe textures in hexagonal lyotropic mesophases," Journal de Physique II, vol. 6, no. 2, pp. 281-303, 1996.

[20] G. Napoli and A. Nobili, "Mechanically induced helfrich-hurault effect in lamellar systems," Physical Review E, vol. 80, no. 3, p. 031710, 2009.

[21] C. A. Whitfield, T. C. Adhyapak, A. Tiribocchi, G. P. Alexander, D. Marenduzzo, and S. Ramaswamy, "Hydrodynamic instabilities in active cholesteric liquid crystals," The European Physical Journal E, vol. 40, no. 4, p. 50, 2017.

[22] K. Harth, T. Trittel, K. May, and R. Stannarius, "Wrinkling of freely floating smectic films," arXiv preprint arXiv:1809.08082, 2018.

[23] A. Rapini and M. Papoular., "Distortion d'une lamelle nématique sous champ magnétique. conditions d'angrage aux paroix," J. Phys. Colloque C4, p. 54, 1969.

[24] T. Ishikawa and O. Lavrentovich, "Dislocation profile in cholesteric finger texture," Physical Review E, vol. 60, no. 5, p. R5037, 1999.

[25] E. A. Brener and V. I. Marchenko, "Nonlinear theory of dislocations in smectic crystals: An exact solution," Phys. Rev. E, vol. 59, pp. R4752-R4753, May 1999.

[26] L. Lejček, V. Novotnía, and M. Glogarová, "A model of field induced stripe texture in the cholesterics with extremely short pitch," Liquid Crystals, vol. 0, no. 0, pp. 1-5, 2018.

[27] V. Novotná, V. Hamplová, M. Glogarová, L. Lejček, and E. Gorecka, "Effect of the applied electric field on new cholesterics with extremely short pitch," Liquid Crystals, vol. 45, no. 4, pp. 634-640, 2018.

[28] G. Napoli, "On smectic-a liquid crystals in an electrostatic field," IMA Journal of Applied Mathematics, vol. 71, no. 1, pp. 34-46, 2006.

[29] R. Fettiplace, D. M. Andrews, and D. A. Haydon, "The thickness, composition and structure of some lipid bilayers and natural membranes," The Journal of Membrane Biology, vol. 5, pp. 277-296, Sep 1971.

[30] S. Hladky and D. Gruen, "Thickness fluctuations in black lipid membranes," Biophysical journal, vol. 38 , no. 3, pp. 251-258, 1982.

[31] E. Evans and W. Rawicz, "Entropy-driven tension and bending elasticity in condensed-fluid membranes," Phys. Rev. Lett., vol. 64, pp. 2094-2097, Apr 1990.

[32] G. Napoli and S. Turzi, "On the determination of nontrivial equilibrium configurations close to a bifurcation point," Computers \& Mathematics with Applications, vol. 55, no. 2, pp. 299 - 306, 2008. Modeling Granularity. 